\title{
Predictors of maternal outcome in women on mechanical ventilation in an obstetric intensive care unit: an observational study
}

\author{
Tanu Soni, Priyadarshini Tiwari*
}

Department of Obstetrics and Gynecology, NSCB Medical College, Jabalpur, Madhya Pradesh, India

Received: 07 December 2018

Accepted: 07 January 2019

\section{*Correspondence:}

Dr. Priyadarshini Tiwari,

E-mail: drpriya2004@yahoo.co.in

Copyright: (C) the author(s), publisher and licensee Medip Academy. This is an open-access article distributed under the terms of the Creative Commons Attribution Non-Commercial License, which permits unrestricted non-commercial use, distribution, and reproduction in any medium, provided the original work is properly cited.

\begin{abstract}
Background: Present study was designed to note the indications for and the complications and outcome of women on mechanical ventilation in our obstetric intensive care unit, and in addition to look for the applicability and correlation of Sequential Organ Failure Assessment (SOFA) scores for the prediction of outcome in these women.

Methods: A prospective observational study was conducted in the obstetric intensive care unit of our teaching hospital which included all women requiring mechanical ventilation in the study period. The diagnosis of the woman on admission, the clinical course and outcome along with total maximum sequential organ failure assessment (SOFA) score and SOFA score for each system were noted. Women were divided into two groups, survivors and nonsurvivors. Student t test and chi square test were used for analysis.

Results: The foremost indication for mechanical ventilation was hypertension in pregnancy namely eclampsia and pre-eclampsia, followed by obstetric hemorrhage and then by hepatic failure. Maternal mortality rose significantly as the number of days of mechanical ventilation increased ( $p$ value <0.05). The total SOFA score correlated highly significantly with the outcome $(\mathrm{p}<0.0001)$.

Conclusions: In women with eclampsia and pre-eclampsia suffering from respiratory failure, survival is inversely correlated with the number of days of mechanical ventilation. The total SOFA score is highly predictive of the woman's outcome and all individual organ system scores also significantly correlate with outcome except for the score of coagulation system.
\end{abstract}

Keywords: Eclampsia, Maternal mortality, Mechanical ventilation, Organ dysfunction scores, Pre-eclampsia

\section{INTRODUCTION}

India is a country having a high maternal mortality rate. ${ }^{1}$ Our teaching hospital is situated in an underdeveloped state of India with a large tribal population. The awareness of antenatal care is still poor in the state, and the maternal mortality is high despite government efforts. There is a high maternal mortality attributable to preventable causes.

Respiratory failure is an important complication in all such patients. Most of these patients require mechanical ventilation which can be given only in specialized intensive care units under supervision of trained intensivist. The outcome of these patients may depend on a number of factors and the mortality is generally high in such patients. Authors have tried to find out what were the conditions which led to respiratory failure in our patients and what were the factors which affected their outcome when they received mechanical ventilation.

It is useful to have a scoring system to predict the outcome of any patient in the ICU. There are a number of scoring systems which are used for prognostication in critically ill women in the ICU. ${ }^{2}$ APACHE I (Acute Physiology and Chronic Health Evaluation I), APACHE 
II, APACHE III and APACHE IV have been devised and modified according to the requirements of critical patients. ${ }^{2,3}$ The APACHE score and SAPS score include many parameters and the use of these scoring systems appears more cumbersome. The SOFA score (Sequential Organ Failure Assessment Score) based on six different scores, one each for the respiratory, cardiovascular, hepatic, coagulation, renal and neurological systems, was initially devised for cases of sepsis, uses less parameters and is easier to use in all critically ill patients. ${ }^{4}$ Authors used the SOFA scoring system in critically ill women requiring mechanical ventilation in our ICU and tried to find out how good it was for predicting the prognosis in them.

\section{METHODS}

Our obstetric ICU was established in August 2014. It is a thirteen bedded facility where residents and nurses are on duty round the clock for the patients. The intensivist is on call and can come over to attend the patient in few minutes. The unit has a turnover of 400 to 500 admissions per year.

A prospective, observational study was conducted among all the women who were put on mechanical ventilation in our ICU in the period from March 2015 to August 2016.The epidemiological characters were studied.

The diagnosis of all women requiring ventilation and the various interventions required during the treatment such as administration of blood components, inotropes, higher antibiotics and need for dialysis was noted. The number of days of mechanical ventilation required in each patient was noted to see whether the duration of ventilation influences the survival of the patient.

The SOFA scores (sequential organ failure assessment scores) of all the women at the time of initiation of mechanical ventilation was noted. All the six components of the SOFA score for various systems namely respiratory, nervous system, cardiovascular, liver function, renal function and coagulation were calculated, and the total SOFA score was also noted. The facilities for Arterial Blood Gas analysis were not available in our hospital in the study period and the analysis was done after sending the samples to a private laboratory, making it difficult to send repeated samples in any of the women. Hence, Authors did not study the maximum SOFA score of any woman during her stay in the ICU. The total SOFA score as well as the individual components of the SOFA score were calculated to study their correlation with the outcome of the women. Higher scores in this system are indicative of progressive severity of dysfunction.

\section{Statistical analysis}

The data was checked and rechecked for any errors and then imported in SPSS version 20. Chi Square Test,
Student $\mathrm{t}$ Test and Wilcoxon Rank Sum (Mann Whitney test) were used for analysis. The SOFA scores for each organ were compared between women who survived and those who did not survive by using the non-parametric Wilcoxon rank sum test.

The predictive efficacy of the SOFA scores for identifying survivors was determined by using statistical tests such as sensitivity, specificity and area under the Receiver Operating Characteristic (ROC) curve. Statistical significance was pre-defined as a value $<0.05$.

\section{RESULTS}

A total of 73 critically ill obstetric patients required mechanical ventilation in our ICU during the study period. Out of all these only $2.7 \%$ were our booked patients and $97.3 \%$ were referred.

They were mostly young with a mean age of $24.7 \pm 4.3$ years with primigravidae being $57.5 \%$ and multigravidae being $42.5 \%$. Out of these women 29 women required assisted ventilation in the antepartum period and 44 were on ventilation in the postpartum period.

Out of the antenatal women 19 underwent caesarean section. 8 women out of these 19 survived. 48 had delivered vaginally either in our institute or referred after delivery. Out of those who delivered vaginally, 23 survived. It was observed that whether the patient delivered vaginally or had a cesarean section, there was no significant difference in survival ( $p>0.05$ ).

Table 1: Diagnosis of women on mechanical ventilation $\mathrm{N}=73$.

\begin{tabular}{|l|l|l|}
\hline Diagnosis & Number & Percentage \\
\hline Eclampsia & 30 & 41.09 \\
\hline Severe Pre-eclampsia & 15 & 20.54 \\
\hline Hypertensive Heart Failure & 2 & 2.73 \\
\hline Dilated cardiomyopathy & 2 & 2.73 \\
\hline Obstetric hemorrhage & & \\
\hline Antepartum hemorrhage & 1 & 1.37 \\
\hline Postpartum hemorrhage & 5 & 6.84 \\
\hline Ruptured uterus & 3 & 4.11 \\
\hline Hepatic failure & 5 & 6.84 \\
\hline Septicemia & 2 & 2.73 \\
\hline DIC & 1 & 1.37 \\
\hline Pulmonary embolism & 2 & 2.73 \\
\hline Swine flu & 2 & 2.73 \\
\hline Anaphylactic reaction & 1 & 1.37 \\
\hline Sicklecell disease & 1 & 1.37 \\
\hline Congenital heart disease & 1 & 1.37 \\
\hline Severe anemia with CCF & 1 & 1.37 \\
\hline
\end{tabular}

2 women aborted. 3 had exploratory laparotomy for ruptured uterus, of whom two were operated in our institute and one was referred to us from the periphery. Of these, only one out of the three who was operated in 
our institution survived. One patient with previous two caesareans with central placenta previa had caesarean section followed by hysterectomy and ligation of internal iliac vessels for PPH survived. One patient died undelivered. The most important diagnosis of women requiring ventilator support was eclampsia in 30 women $(41.1 \%)$ followed by severe pre-eclampsia in $15(20.5 \%)$ women. (Table 1).

On analyzing the duration of ventilation majority of the women i.e. $32(41.1 \%)$ were on ventilator support for 1-2 days Figure 1. Two peaks of high mortality were seen, one in the first two groups up to 2 days of ventilation and the other in the last group requiring 5 days or more of ventilation (Figure 1).

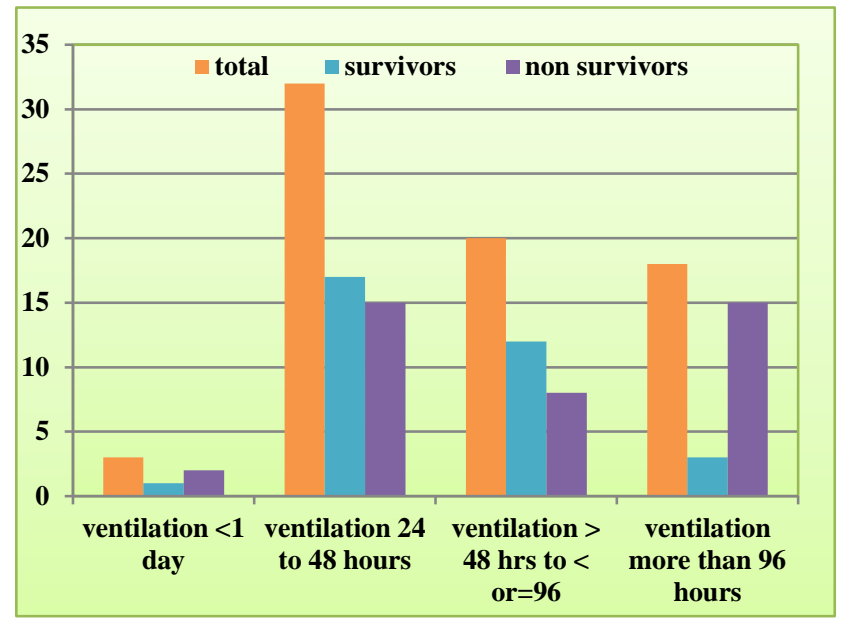

Figure 1: Survival against the duration of ventilation

Mean duration of ventilation among non-survivors was 4.38 days and 2.61 days among survivors ( $p<0.05$ ). Complications occurred in 42 women during ventilator support Table 2 .
Table 2: Complications in patients on mechanical ventilation.

\begin{tabular}{|l|l|}
\hline Complications & $\begin{array}{l}\text { Total } \\
\text { frequency (\%) }\end{array}$ \\
\hline Failure of three or more organ systems & $17(23.2)$ \\
\hline Renal failure & $7(9.5)$ \\
\hline Hypoxic convulsions & $4(5.4)$ \\
\hline Blood Transfusion reaction & $1(1.3)$ \\
\hline Culture positive Septicaemia & $9(12.3)$ \\
\hline Secondary PPH & $1(1.3)$ \\
\hline Arrhythmias & $1(1.3)$ \\
\hline
\end{tabular}

The most common complication was multiorgan failure in 17 women followed by septicemia in 9 and renal failure in 7. Recurrent seizures occurred in 4 and Posterior Reversible Encephalopathy Syndrome (PRES) was seen on neuroimaging in 2 women of eclampsia, though all women of eclampsia were not subjected to neuroimaging studies. Blood transfusion reaction, cardiac arrhythmias and secondary PPH were seen in 1 case each. Multiorgan failure contributed to $42.5 \%$ of mortality.

The mean total SOFA scores in our patients were calculated in both survivors and non-survivors. In present study the mean total SOFA score in non-survivors was $10.50(+3.679 \mathrm{SD})$ and that in survivors was $6.30(+3.197 \mathrm{SD})$. The total mean score was $8.60(+4.037$ $\mathrm{SD})$. Comparison of mean SOFA scores of each system between survivors and non survivors showed a statistically significant difference in the respiratory, nervous, cardiovascular, hepatic and renal system $(\mathrm{p}<0.05)$. The $\mathrm{p}$ values for each system were respiratory $0.002 \quad(<0.05)$, nervous system $0.029 \quad(<0.05)$, cardiovascular system $0.002(<0.05)$, hepatic $0.003(<$ $0.05)$ and renal $0.017 \quad(<0.05)$. The scores in the coagulation system did not show a significant difference, $\mathrm{p}=0.221(\mathrm{p}>0.05)$ (not significant) (Table 3$)$.

Table 3: Comparison of mean sofa scores between survivors and non-survivors.

\begin{tabular}{|l|l|l|l|l|}
\hline \multirow{2}{*}{ Organ/system evaluated } & \multicolumn{2}{c}{ Mean $(\mathrm{SD})$ SOFA score } & Total (Mean \pm SD) & \multirow{2}{*}{ P-value } \\
\cline { 2 - 5 } & Non-Survivor & Survivor & & 0.002 \\
\hline Respiratory & $3.47(0.59)$ & $2.91(0.87)$ & $3.22(0.79)$ & 0.029 \\
\hline CVS & $1.78(1.77)$ & $0.94(1.39)$ & $1.40(1.66)$ & 0.002 \\
\hline Hepatic system & $1.85(1.79)$ & $0.61(1.14)$ & $1.29(1.65)$ & 0.003 \\
\hline Coagulation & $1.22(1.20)$ & $0.48(0.83)$ & $0.89(1.11)$ & 0.221 \\
\hline Renal System & $1.35(1.33)$ & $0.97(1.18)$ & $1.18(1.27)$ & 0.017 \\
\hline Total SOFA Score & $0.83(0.90)$ & $0.39(0.65)$ & $0.63(0.83)$ & 0.000 \\
\hline
\end{tabular}

The total SOFA score showed a highly significant difference in the two groups with $\mathrm{p}<0.0001$ which is highly significant. The total SOFA score of $>7$ showed a sensitivity of $80.00 \%$ (CI; 64.4, 90.9) and specificity of
$72.73 \%$ (CI; 54.5, 86.7). At the total SOFA score of $>9$ sensitivity was $65.00 \%(\mathrm{CI} ; 48.3,79.4)$ and specificity was $84.85 \%$ (CI; 68.1, 94.9) for mortality (Table 4). 
Table 4: Sensitivity, specificity and area under receiver operator characteristic.

\begin{tabular}{|l|l|l|l|}
\hline $\begin{array}{l}\text { SOFA } \\
\text { score }\end{array}$ & Sensitivity & Specificity & $\begin{array}{c}\text { Area under the } \\
\text { (ROC) curve }\end{array}$ \\
\hline$\geq 2$ & 100.00 & 0.00 & \\
\hline$>3$ & 97.50 & 18.18 & \\
\hline$>4$ & 95.00 & 33.33 & \\
\hline$>5$ & 87.50 & 51.52 & \\
\hline$>6$ & 87.50 & 63.64 & \\
\hline$>7$ & 80.00 & 72.73 & \\
\hline$>8$ & 70.00 & 81.82 & \\
\hline$>9$ & 65.00 & 84.85 & \multirow{2}{*}{$0.82(95 \%$} \\
\hline$>10$ & 50.00 & 90.91 & \multirow{2}{*}{ CI; $0.710-0.916)$} \\
\hline$>11$ & 37.50 & 90.91 & \\
\hline$>12$ & 25.00 & 90.91 & \\
\hline$>13$ & 20.00 & 93.94 & \\
\hline$>14$ & 17.50 & 96.97 & \\
\hline$>15$ & 5.00 & 100.00 & \\
\hline$>17$ & 2.50 & 100.00 & \\
\hline$>20$ & 0.00 & 100.00 & \\
\hline & & & \\
\hline
\end{tabular}

The accuracy for the total SOFA score of $>7$ was $76.71 \%$ and the accuracy for the total SOFA score of $>9$ was $73.97 \%$. The area under the ROC curves for the total maximum SOFA scores was 0.813 with $95 \%$ confidence interval (0.704-0.895) (Figure 2).

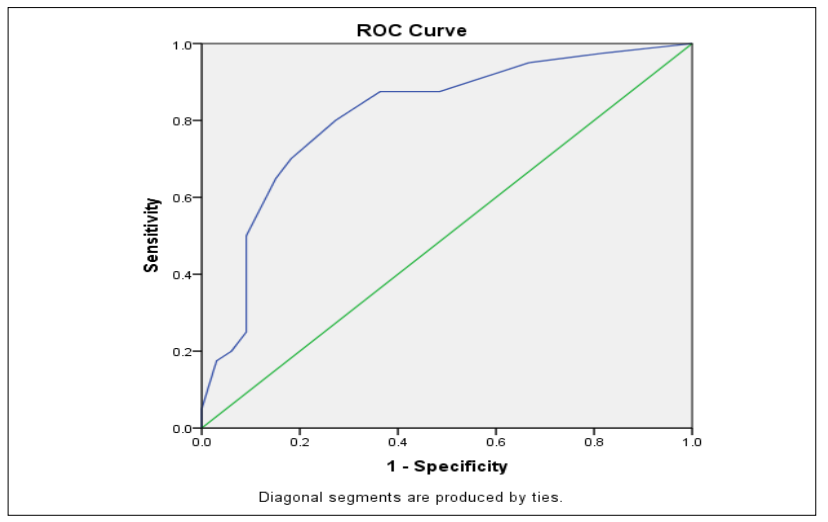

Figure 2: ROC curve for total SOFA scores.

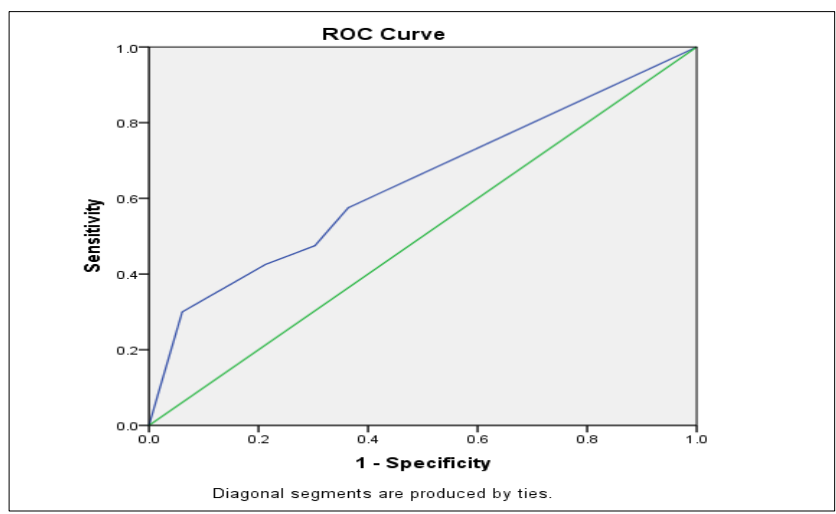

Figure 3: ROC curve for the nervous system AUC 0.638 .
On calculating the maximum SOFA scores for each organ system 95\% confidence intervals around the point estimates for the area under ROC curves showed that the upper limits were $>0.75$ for all systems except coagulation (Figure 2).

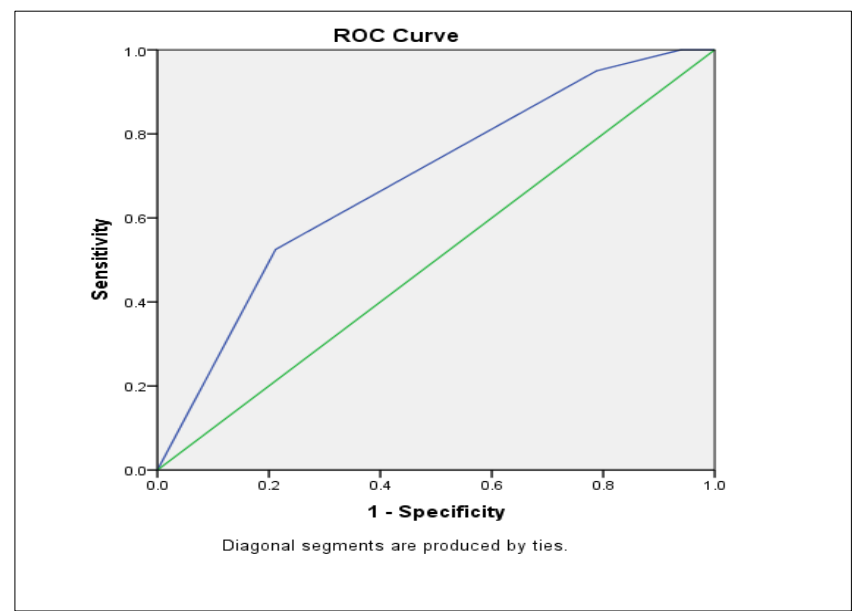

Figure 4: ROC curve for the respiratory system AUC 0.689.

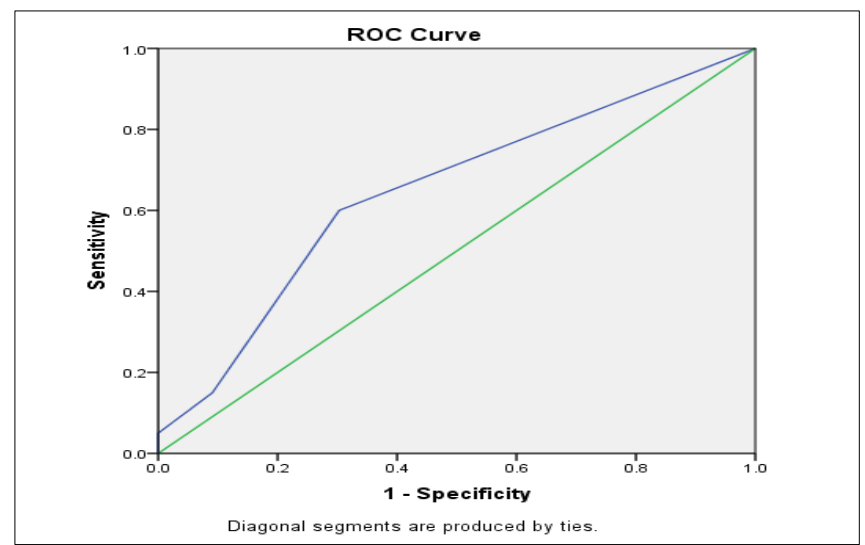

Figure 5: ROC curve for the excretory system AUC 0.646.

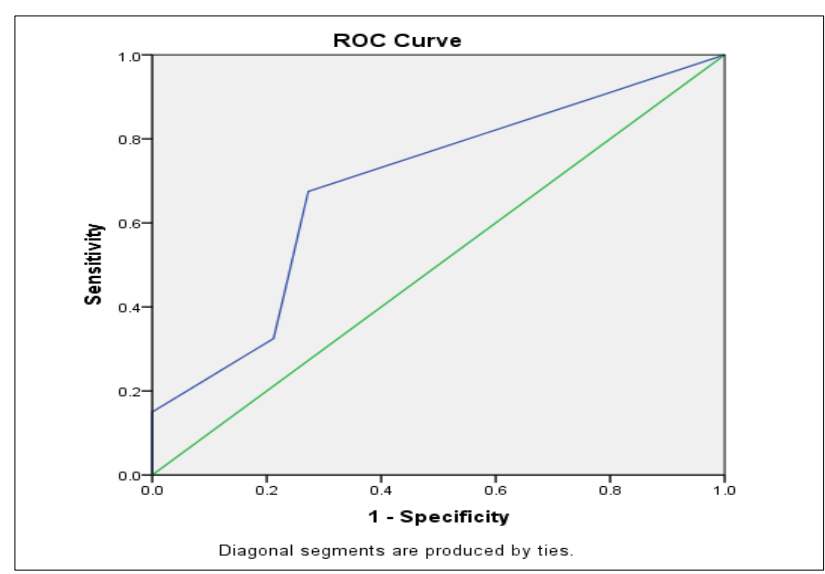

Figure 6: ROC curve for the hepatic system AUC 0.69. 


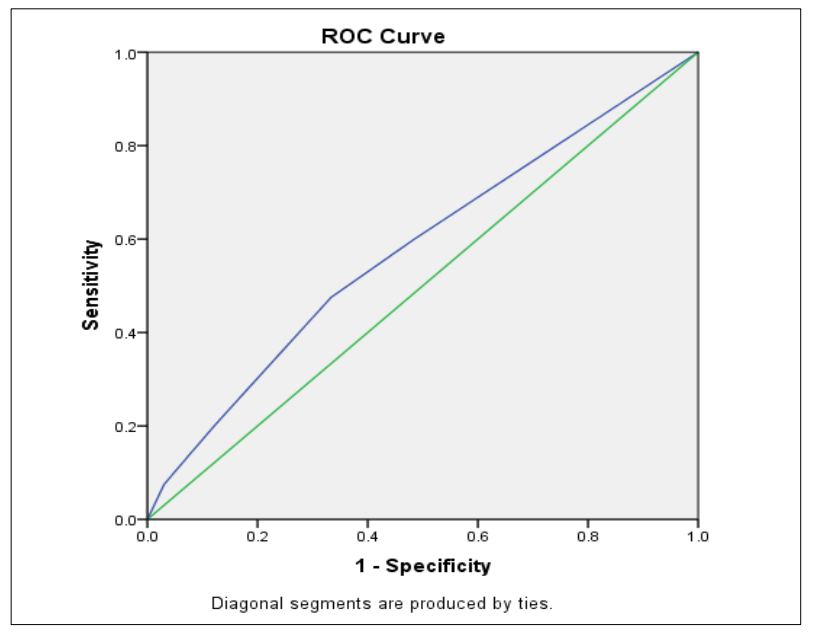

Figure 7: ROC curve for coagulation AUC 0.579.

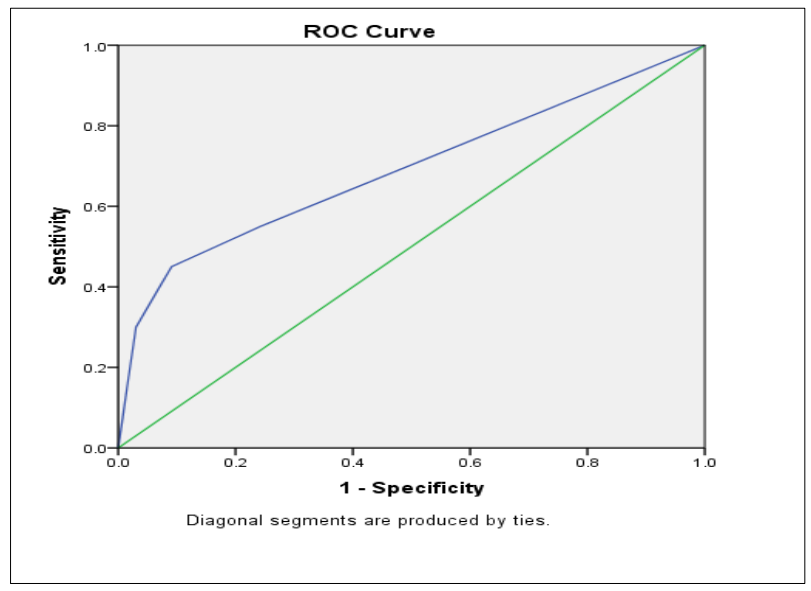

Figure 8: ROC curve for the cardiovascular system AUC 0.69.

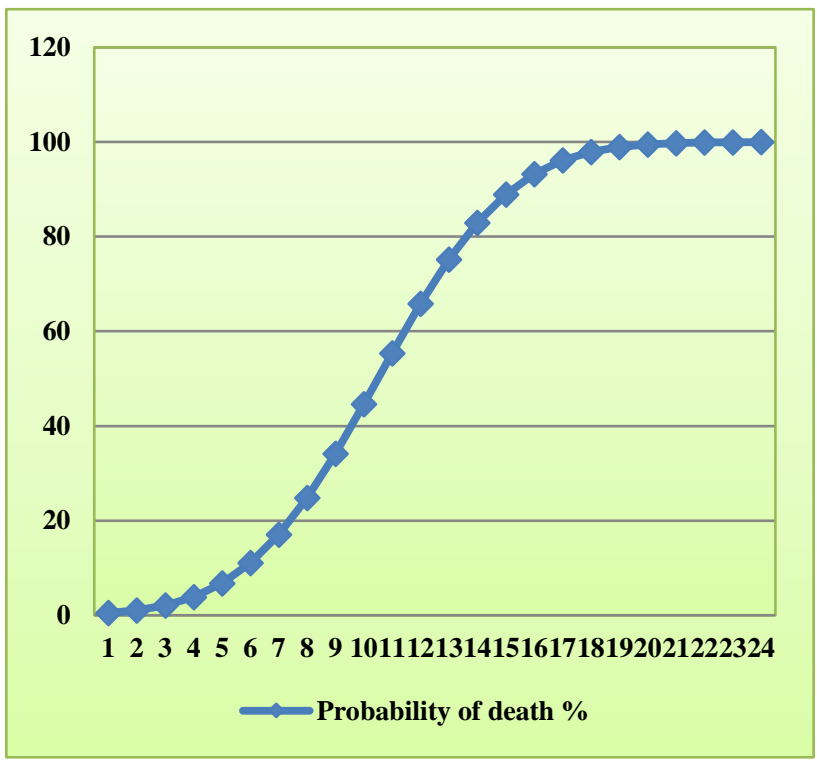

Figure 9: Estimated probability of death of women on mechanical ventilation, for each value of the total SOFA score.
The probability of death increases with the increasing SOFA score with $80-90 \%$ chances of mortality with scores > 14 (Figure 8).

\section{DISCUSSION}

The most important indication requiring mechanical ventilation in our ICU was eclampsia and severe preeclampsia namely hypertensive disorders of pregnancy. Out of 30 women with eclampsia 7 were complicated by HELLP syndrome. Similar finding was also seen in other studies where eclampsia and severe pre-eclampsia were the most important indication for admission in the obstetric ICU. ${ }^{5}$

The cause of respiratory failure in these women is generally ARDS but sometimes these women may also have pulmonary edema due to left ventricular failure. Heart failure in these women may be caused by either hypertensive heart failure of pregnancy (HHFP) or peripartum cardiomyopathy (PPCM). ${ }^{6}$ ARDS was defined as the acute onset of respiratory failure, bilateral infiltrates on chest radiograph, hypoxemia as defined by a $\mathrm{PaO} 2 / \mathrm{FiO} 2$ ratio $\leq 200 \mathrm{mmHg}$, and no evidence of left atrial hypertension or a pulmonary capillary pressure $<18$ $\mathrm{mmHg}$ (if measured) to rule out cardiogenic edema. ${ }^{7}$ The diagnosis of ARDS versus heart failure can be confirmed with the findings on x-ray chest and echocardiography. Women are more predisposed to ARDS during pregnancy due to the physiological changes that accompany pregnancy and show a good response to treatment. ${ }^{7}$

Obstetric hemorrhage was the next important indication for assisted ventilation. Out of a total of 9 women five had PPH, 3 had uterine rupture and 1 had abruption. Out of the women with PPH one was a case of previous two cesareans with central placenta previa who had massive PPH in the operation theatre and had hysterectomy with ligation of internal iliac vessels. Apart from this patient there were four women with PPH referred very late from the periphery in irreversible shock out of which only one survived. There were 3 women with uterine rupture out of whom two survived and 1 died. In the two survivors one was a case of scar rupture and the other was rupture following obstructed labor. The patient who died was a primigravidae with rupture of the uterus probably due to injudicious administration of oxytocics in the periphery and was complicated by septicemia and irreversible shock. One case of abruption required ventilation but did not survive. A study from Kerala documented PPH as the foremost diagnosis requiring ventilation with $45.3 \%$ women of PPH with $16 \%$ women complicated with DIC. ${ }^{8}$

Surprisingly hepatic failure complicating pregnancy was the next important cause in women requiring ventilation. 5 women $(6.8 \%)$ required ventilation and 4 died which was $10 \%$ of the total deaths in the study. Of the 4 women who died, one had acute fatty liver of pregnancy and the rest had viral hepatitis. Acute fatty liver usually presents with moderately elevated liver enzymes, hypoglycemia 
and coagulopathy. Viral hepatitis usually presents with remarkably high liver enzymes and positive serology without hypoglycemia. $^{9}$

Acute heart failure was the next (3 women, $4.1 \%$ ) in requiring ventilation. Out of these, one woman had failure in the antepartum period and two in the postpartum period. Hypertensive Heart Failure of Pregnancy (HHFP) and Peripartum Cardiomyopathy (PPCM) are two varieties of cardiac failure seen in pregnancy. ${ }^{6}$ HHFP is more commonly associated with PIH and history of hypertension and manifests in the antepartum period. PPCM is more likely to manifest postpartum with cardiomegaly, cardiac thrombus, pulmonary hypertension and low ejection fraction below $35 \%$. Though the treatment of both conditions is similar, the prognosis of PPCM is graver. ${ }^{6}$ Pre-eclampsia is associated with PPCM. ${ }^{6}$ All three had an uneventful recovery and none had any evidence of dilated cardiomyopathy on $\mathrm{x}^{-}$ray chest suggesting the probability of HHFP in all three women. In 2015 a number of women were suspected to have H1N1 flu but only two were swab positive and required ventilator support. Out of these one died and the other survived. One woman with post abortal sepsis required ventilation. This was a case of a young woman who had an illegal abortion in a remote village and survived after management in the ICU.

On trying to correlate the mortality with the duration of ventilation two peaks of mortality were seen. The first peak was seen in the first two days of ventilation and the next peak was seen in women requiring 5 days or more of mechanical ventilation. The first peak of mortality was seen because of referrals coming to the ICU in very poor condition mostly with multiorgan failure. The second peak was seen in 5 days or more of ventilation. The women who require ventilation for 5 days or more are susceptible to severe nosocomial infections and the sequel of sepsis.

When the mean duration of ventilation was observed between survivors and non-survivors the survivors had a mean duration of 2.61 days as compared to 4.38 days in non-survivors showing that the duration of ventilation was significantly higher in non-survivors $(p<0.05)$. The best prognosis was observed in those women who received ventilation for a short period i.e. less than 4 days in which the more grave complications were prevented.

Complications occurred in 42 women on ventilator support most of which were related to underlying pathophysiology of the ongoing disease process. The prognosis of women on mechanical ventilation depends on

- The condition of the patient at the time of initiation of MV and

- The complications developed during the course of MV. The common complications were ventilator associated pneumonias, septicemia and ARDS (Table 2).

Culture positive septicemia was seen in 9 women out of whom 5 died and 4 survived. Failure of three or more organ systems like hepatic, renal, coagulation, cardiovascular occurred in 17 women resulting in death of all these women (Table 2). Eclampsia and severe preeclampsia are disorders characterized by multiorgan involvement. Oxidative stress leading to endothelial injury and ischemia as the basic pathophysiology of preeclampsia and in association with ventilator associated pneumonias and septicemia on MV lead to the affection of multiple organs which may lead to multiorgan failure in critically ill women. 7 women (Table 2) had renal failure out of which 4 died and 3 survived. These were women who had failure of three or more organ systems along with septicemia.

Rest of the women had sporadic complications like hypoxic seizures in 4 women, transfusion reaction in 1 patient, secondary PPH in 1, arrhythmias in 1 and PRES in 2 women (Table 2). PRES (posterior reversible encephalopathy syndrome) is a common condition seen in eclampsia and as the name suggests it is reversible. ${ }^{10}$ All women with eclampsia were not subjected to cranial imaging and this is not representative of the true incidence of the condition. Present study showed a high maternal mortality. Out of 73 total women 40 (54.8\%) died and $33(45.2 \%)$ survived.

In present study the mean total SOFA score in nonsurvivors was $10.50(+3.679 \mathrm{SD})$ and that in survivors was $6.30(+3.197 \mathrm{SD})$. The total mean score was $8.60(+$ $4.037 \mathrm{SD})$. There was a statistically highly significant difference between the total SOFA scores of the two groups (Mann Whitney test $) \quad(\mathrm{p}<0.0001$, highly significant).

Mean scores of all the individual systems except for coagulation showed a significant difference in the SOFA scores between survivors and non-survivors (Table 3). Since the parameter of coagulation taken in the study was platelet count which could have been affected by the transitory nature of thrombocytopenia in pregnancy in general and hypertensive disorders and HELLP syndrome in particular which resolves quickly following delivery and may not be a very reliable marker to predict the prognosis of the woman. Similar results were found in other studies where coagulation failure $(<50,000)$ did not significantly affect the prognosis of the patient. ${ }^{11,12}$

The use of the total SOFA scores was found to be a good indicator of prognosis in this population with a cut off value $>7$ at which point the sensitivity was $80.00 \%$ and specificity was $72.73 \%$ which significantly increased the probability of maternal deaths in women on ventilator. In a study conducted on all women admitted in ICU the cut off value was $>6$ with a sensitivity of 88.9 and specificity 91.113.Present study included only those women who 
were on mechanical ventilation and this could have been one of the causes of different cut off values in the scores. The area under the ROC curve for total SOFA scores was 0.958 as compared to 0.82 in present study. ${ }^{13}$ Also, the area under the ROC curve for coagulation system was 0.728 as compared to 0.579 in present study. ${ }^{13}$ In a study conducted on obstetric patients in South India, an accuracy for mortality was observed to be $94.20 \%$ at the score of $>10$ and $95.65 \%$ at the score of $>12$ in the total SOFA scores. ${ }^{12}$ In present study accuracy at the score of $>7$ was $76.71 \%$ and at the value of $>9$ it was $73.97 \%$. In the above-mentioned study, the area under ROC curve for total SOFA scores was 0.77 as compared to 0.82 in ours. ${ }^{12}$ The area under ROC for coagulation was 0.46 as against 0.579 in present study and the $p$ value for coagulation was 0.73 in this study as against 0.221 in ours which was not significant in both studies. ${ }^{12}$ The $p$ value for coagulation system was not significant in this study, similar to our result. The reason for this insignificant $p$ value in the coagulation system appears to be the parameter used for this system which was the platelet count. The platelet counts are affected by the physiological changes of pregnancy. Present study population had the highest number of admissions for eclampsia and severe pre-eclampsia including HELLP syndrome where the platelet counts are affected by the disease pathology, but the recovery of the thrombocytopenia is quick following delivery. Also, the platelet count in itself may not be indicative of the quality and function of the platelets. In an Indian prospective study of obstetric patients admitted to the ICU, the total SOFA score on admission displayed an area under the ROC curve was 0.949 against 0.82 in present study. ${ }^{11}$ At a cutoff value of 8.5 , a sensitivity of $86.7 \%$ and specificity of $90.0 \%$ was observed in this study. ${ }^{11}$

The probability of death increases with the increasing SOFA score with 80 to $90 \%$ chances of mortality with scores above (Figure 9). ${ }^{14}$ The cutoff value of the SOFA scores in a prospective study in an Indian ICU was 8.511 while it was $>7$ in present study. This could be interpreted as greater likelihood of mortality at a lower score in present study population as our population comprised of all women having respiratory failure and were having a higher score for the respiratory system with greater susceptibility for nosocomial infections such as ventilator associated pneumonias and septicemia. The other studies have reported greater areas under the ROC curves for total SOFA scores as compared to ours. These studies are retrospective studies where the maximum SOFA score for any individual organ system during the entire course of the woman's stay in the ICU was taken for calculation. Thus, the maximum SOFA scores for each system were the poorest scores for each variable recorded during the entire period of the woman's ICU admission. Hence here the total maximum SOFA score was not an indicator of the woman's condition on any particular day. Present study was a prospective study where the SOFA score on the initiation of mechanical ventilation was calculated for predicting the prognosis and differed in the methodology in a number of ways. In a similar prospective study from India, the total SOFA score on admission in all obstetric cases admitted to the ICU was found to be excellent for prediction of the prognosis. ${ }^{11}$ This study was published after the completion of present study period and dealt with all obstetric admissions and not the women on mechanical ventilation. In this study the total maximum SOFA scores were calculated in a way similar to a retrospective study by Oliveira-Neto A which did not represent the woman's condition on any particular day. ${ }^{11,13}$

Strengths of present study: Present study is the first study in which patients on mechanical ventilation were studied along with the utility of the SOFA scoring system for prediction of the prognosis in obstetric cases at the initiation of mechanical ventilation.

Weakness of present study: due to the constraints in getting the blood gas analyses in our institution, the SOFA score could be calculated only at the time of initiation of mechanical ventilation. For this reason, the change in the SOFA scores during admission and the maximum SOFA scores could not be done.

\section{CONCLUSION}

Hypertensive disorders of pregnancy are the foremost diagnosis requiring mechanical ventilation in our ICU. The likelihood of survival is inversely correlated with the number of days of mechanical ventilation. The total SOFA scores are excellent in predicting the prognosis of women on ventilation on admission in the obstetric ICU with a cutoff score of 7 at which there is a low probability of mortality. At a score of 9 and above, the likelihood of death increases progressively with nearly $100 \%$ chances of mortality in a score of 15 and above. The individual score for the coagulation system did not have a good prognostic value and the authors recommend further studies to look for the inclusion of better applicable parameters such as INR or fibrinogen levels for coagulation to modify the SOFA scores in obstetrics.

\section{ACKNOWLEDGMENTS}

Authors would like to thank Dr. Neeraj Narang for his work as an intensivist in taking care of our critical patients on mechanical ventilation in the ICU.

Funding: No funding sources

Conflict of interest: None declared

Ethical approval: The study was approved by the Institutional Ethics Committee

\section{REFERENCES}

1. Hogan MC, Foreman KJ, Naghavi M, Ahn SY, Wang M, Makela SM, Lopez AD, Lozano R, Murray CJ. Maternal mortality for 181 countries, 19802008: a systematic analysis of progress towards 
Millennium Development Goal 5. The Lancet. 2010;375(9726):1609-23.

2. Vincent JL, Moreno R. Clinical review: scoring systems in the critically ill. Critical care. 2010; 14(2):207.

3. Zimmerman JE, Kramer AA, McNair DS, Malila FM. Acute Physiology and Chronic Health Evaluation (APACHE) IV: hospital mortality assessment for today's critically ill patients. Crit Care Med. 2006;34(5):1297-310.

4. Vincent JL, De Mendonça A, Cantraine F, Moreno R, Takala J, Suter PM, Sprung CL, Colardyn F, Blecher S. Use of the SOFA score to assess the incidence of organ dysfunction/failure in intensive care units: results of a multicenter, prospective study. Criti Care Med. 1998;26(11):1793-800.

5. Mowafy AH, Mashhour KS. Clinical Characteristics and Outcomes of Obstetric Patients Requiring ICU Admission. Med J Cairo Univers. 2010;78(2).

6. Ntusi NB, Badri M, Gumedze F, Sliwa K, Mayosi BM. Pregnancy-associated heart failure: a comparison of clinical presentation and outcome between hypertensive heart failure of pregnancy and idiopathic peripartum cardiomyopathy. PLoS One. 2015;10(8):e0133466.

7. Catanzarite V, Willms D, Wong D, Landers C, Cousins L, Schrimmer D. Acute respiratory distress syndrome in pregnancy and the puerperium: causes, courses, and outcomes. Obstet Gynecol. 2001;97(5):760-4.

8. Sreenivas KS, Beevi AN, Devi NU. Clinical Characteristics and Outcome of Obstetric Patients
Who Required Mechanical Ventilation in a Tertiary Care Hospital in North Kerala. Indian $\mathbf{J}$ Clinic Prac.2014;25(6):552-7.

9. Acharya SK. Hepatitis E and acute liver failure in pregnancy. J Clinic Experiment Hepatol. 2013;3(3):213-24.

10. Fugate JE, Rabinstein AA. Posterior reversible encephalopathy syndrome: clinical and radiological manifestations, pathophysiology, and outstanding questions. The Lancet Neurol. 2015;14(9):914-25.

11. Jain S, Guleria K, Suneja A, Vaid NB, Ahuja S. Use of the Sequential Organ Failure Assessment score for evaluating outcome among obstetric patients admitted to the intensive care unit. Int $\mathbf{J}$ Gynecol Obstet. 2016;132(3):332-6.

12. Kallur SD, Bada VP, Reddy P, Pandya S, Nirmalan PK. Organ dysfunction and organ failure as predictors of outcomes of severe maternal morbidity in an Obstetric Intensive Care Unit. J Clinic Diagnos Res: JCDR. 2014;8(4):OC06.

13. Oliveira-Neto A, Parpinelli MA, Cecatti JG, Souza JP, Sousa MH. Sequential organ failure assessment score for evaluating organ failure and outcome of severe maternal morbidity in obstetric intensive care. Scientific World J. 2012; 2012:172145.

Cite this article as: Soni T, Tiwari P. Predictors of maternal outcome in women on mechanical ventilation in an obstetric intensive care unit: an observational study. Int J Reprod Contracept Obstet Gynecol 2019;8:721-8. 